\title{
Development of the Knowledge of Genome Sequencing (KOGS) Questionnaire
}

Running title: Knowledge of Genome Sequencing (KOGS)

Saskia C Sanderson $\mathrm{PhD}^{1,2}$, Bao Sheng Loe MPhil ${ }^{3}$, Lyn Chitty $\mathrm{MD}^{1}$, Maddie Freeman $\mathrm{MSc}^{2}$, Camila Gabriel $\mathrm{MSc}^{4}$, Danielle Stevenson $\mathrm{PhD}^{1}$, Chris Gibbons $\mathrm{PhD}^{3}$, Celine Lewis $\mathrm{PhD}^{1}$

${ }^{1}$ North East Thames Regional Genetics Services, Great Ormond Street Hospital, London, UK

${ }^{2}$ Department of Behavioural Science and Health, University College London, London, UK

${ }^{3}$ The Psychometrics Centre, University of Cambridge, Cambridge, UK

${ }^{4}$ Center for Bioethics, Harvard Medical School, Boston, USA

Corresponding author at: Saskia Sanderson, North East Thames Regional Genetics Services, Great Ormond Street Hospital, London, UK. Tel: 02078298653 Email: saskia.sanderson@ucl.ac.uk 


\section{ABSTRACT}

Objective: Whole-genome sequencing is being implemented in research and clinical care, yet tools to assess patients' knowledge are lacking. Our aim was to develop a robust measure of whole-genome sequencing knowledge suitable for patients and other stakeholders including research participants, public, students, and health professionals. Methods: An initial set of 17 items was developed via an iterative process including literature review, expert consultation, focus groups, and cognitive interviews with patients, and then administered to 243 individuals. We used exploratory factor analysis and itemresponse theory to confirm the psychometric suitability of the candidate items for assessing whole-genome sequencing knowledge.

Results: There was a strong main component after removing 5 items with low factor loadings. Item and scale homogeneity was achieved using Mokken scale analysis. Three further items were removed because they were misfits, inverse duplicates or resulted in local dependency. The remaining nine items fitted the two-parameter logistic IRT model which achieved excellent fit to the observed data. Cronbach's alpha was 0.79 indicating acceptable reliability.

Conclusion: The KOGS, developed using a rigorous psychometric approach, is a brief and reliable tool.

Practice implications: The KOGS may prove a useful tool for researchers and clinicians using whole-genome sequencing with patients and other stakeholders.

Key words: item response theory, measurement instrument, patient education, patient knowledge, whole-genome sequencing 


\section{INTRODUCTION}

Whole-genome sequencing is gradually being incorporated widely into research and clinical care. For whole-genome sequencing to be integrated effectively and safely it is important that individuals make informed choices before consenting. For informed decision-making there are several patient-reported factors, ${ }^{1}$ of which knowledge is a central component. ${ }^{2}$ One current measure of knowledge of whole-genome sequencing was developed by investigators in the US and administered to 311 patients participating in the ClinSeq project. ${ }^{3}$ This 11-item knowledge measure has been used in several studies with patients and research participants since its development. ${ }^{4}$ However, a different factor structure and low scale-reliability was found for the scale when it was administered to 862 online survey respondents, raising questions about the measure's validity and reliability in different populations and methods of administration. ${ }^{5}$ Recently one additional measure of genomic sequencing knowledge has been developed: the University of North Carolina Genomic Knowledge Scale (UNC-GKS), ${ }^{6}$ but this includes general genetics concepts that have been included in many previous measures of genetics knowledge,$^{7-9}$ with just a small subset specifically on exome sequencing (not whole-genome sequencing). Thus there is a need for a valid, reliable measure of knowledge of whole-genome sequencing that can be used with individuals in a range of settings.

As part of a study (NIHR PG-PB-1014-350160) to measure and examine informed choice among patients being offered whole-genome sequencing via the 100,000 Genomes Project, ${ }^{10}$ we are developing a new measure of informed choice for individuals making decisions about whole-genome sequencing in the context of rare disease diagnosis. During development of the informed choice measure, it became apparent that the majority of the items (questions) being developed were 'context-specific,' i.e. were relevant primarily to the specific context of whole-genome sequencing for the purposes of diagnosing a rare disease. A subset of the items, however, was 'context-neutral', i.e. the items could be administered to 
patients or other stakeholders (e.g. health care providers, students, general public) regardless of the clinical or other context. We therefore expanded our work with patients to develop a new measure of whole-genome sequencing knowledge that could be administered to a range of stakeholders including public, students, and health professionals as well as patients and research participants.

\section{MATERIALS AND METHODS}

\section{Selection of knowledge domains}

In order to identify the domains to include in the knowledge measure overall, and the specific sub-domains (concepts) to cover within each domain we reviewed the domains covered in (a) professional guidelines and recommendations; (b) patient information materials; and (c) an existing measure. ${ }^{3}$ The professional guidelines and recommendations included in the review were: (1) Genetic Alliance UK's 2015 whole-genome sequencing rare diseases patient charter ${ }^{11}$; (2) the British Society of Genetic Medicine's 2013 document on the 100,000 Genomes Project strategy ${ }^{12}$; (3) and a published review and recommendations on whole genome-sequencing informed consent content. ${ }^{13}$ The patient information materials reviewed were (4) the patient information sheet (the version for parents of children with a rare disease) and (5) the consent form for the 100,000 Genomes Project. ${ }^{14}$

This process produced an initial $14 \mathrm{draft}$ domains, which were refined to eight draft domains (and draft sub-domains within each domain) using an iterative process involving two focus groups at two sites with a total of nine health professionals involved in recruiting and consenting patients into the 100,000 Genomes Project, and an advisory board meeting with five board members including a patient (100,000 Genomes Project participant panel member). The eight draft domains produced at the end of this stage of the process were: (1) what is involved in having whole-genome sequencing done (including 'what is a genome'); (2) the purpose; (3) the benefits; (4) the risks; (5) the limitations and uncertainties; (6) how 
the samples and data will be stored and who will have access; (7) how secondary findings will be managed; (8) that it is voluntary. While most of these draft domains described features of whole-genome sequencing that were necessarily context-specific (such as the purpose, and how the samples and data will be stored), two domains could be defined in a context-neutral as well as context-specific way: what is involved in having whole-genome sequencing done (including 'what is a genome'), and the limitations and uncertainties of whole-genome sequencing.

\section{Item development}

A large pool of over 70 candidate items was developed to cover each draft domain using three approaches: (1) review of items included in published measures; (2) items suggested from in-depth qualitative interviews with 20 patients who had consented to take part in the 100,000 Genomes Project (manuscript in preparation); and (3) the Genomic England 100,000 Genomes Project patient information sheet and consent form documents. ${ }^{14}$ As noted above, it became clear at this stage of item development that it would be useful to develop two sets of candidate items for two of the draft domains (what is involved, and limitations and uncertainties): first, a set that were 'context-neutral', i.e. could be administered to patients or other stakeholders regardless of the clinical or other context; and second, a set that were 'context-specific', i.e. that were relevant primarily to a specific clinical context such as whole-genome sequencing for the purposes of diagnosing a rare disease. This paper focuses on the context-neutral items only; the context-specific items will be presented elsewhere.

Based on the literature review, qualitative interviews, information sheets and consent documents, we developed two versions (one true, one false) of 11 items that were 'contextneutral', i.e. 22 items in total. The 22 items covered two draft domains: (1) what is (involved in) whole-genome sequencing, including what is a genome; and (2) limitations and 
uncertainties of whole-genome sequencing. To obtain feedback on the 22 items cognitive interviews were conducted with 4 health professionals (two each at two hospital sites) consenting patients for the 100,000 Genomes Project. The 22 items were also administered to a convenience sample of five non-clinical genetics experts (bioinformaticians) and to a convenience sample of 30 medical students before and after a genetics lecture. Based on the feedback from these different groups, five items were deleted because they were ambiguous or difficult to understand, leaving a total of 17 items. Thirty health care providers (trainee GPs, general practitioners) then completed the 17-item questionnaire. Finally, four cognitive interviews were conducted with patients in the process of consenting to the 100,000 Genomes Project: a think-aloud methodology was used in which the patients went through a questionnaire which included the 17-items, and gave feedback on wording, comprehension, and question order. Revisions were made in light of the feedback and piloting with the various stakeholder groups.

\section{Questionnaire administration}

A written questionnaire comprising the 17 context-neutral candidate items was administered in-person to students and healthcare providers before and after attending lectures on genomics, and university staff via an online survey. The 17 items were also administered to patients after giving consent to have whole-genome sequencing done as part of the 100,000 Genomes Project.

\section{Psychometric and statistical analyses}

\subsection{Psychometric analyses}

Analysis of dimensionality and item properties was conducted in a sequential fashion entailing the following psychometric models: exploratory factor analysis, mokken analysis 
and two-parameter logistic IRT model using the R Statistical Programming Language. Further details on the analysis can be found elsewhere. ${ }^{15}$

We used exploratory factor analysis (EFA) to assess the dimensionality of the nascent scale. The factor structure was analysed using the minimum residual solution with oblique rotation as response options were dichotomous. The tetrachoric correlation matrix was conducted instead of the Pearson correlation in evaluating the presence of latent factors under the assumption of a normal bivariate distribution. ${ }^{16,17}$ Kaiser-Meyer-Olkin (KMO) was employed as a measure of sampling adequacy concerning the assessment of the correlation matrix. Items achieving an index value of 0.80 and above were considered suitable for factor analysis. ${ }^{18}$ Parallel analysis (PA) was used as criteria for retention of factors. Data correlation matrices $(n=1000)$ of random simulated numbers and random resampled numbers that match the number of cases and variables in the observed data were created and compared to PA. Factors from the real eigenvalues that exceeded the average simulated eigenvalues were retained for further analysis. ${ }^{19}$

We used both Mokken analysis and parametric item response theory (IRT) to assess the psychometric properties of the scale. Mokken analysis is a probabilistic (non-parametric) extension of the Guttman scale analysis ${ }^{20}$ that automatically selects items which partition the set of ordinal variables into scales and evaluates the model goodness of fit ${ }^{21}$. Scalability coefficients (also known Loevinger's H coefficients), were provided for the individual items $\left(\mathrm{H}_{\mathrm{i}}\right)$ and the scale $(\mathrm{H})$ as a whole to indicate the strength of the scale as being unidimensional. The $\mathrm{H}$ coefficient threshold was set at 0.30 for both item and scale levels.

IRT was used to model the probabilistic relationship between participants' responses to an item and their level on the construct measured by the scale, and provided information on item discrimination and item difficulty. ${ }^{22}$ Both the item difficulty ( $b$ - location) and the item discrimination (a - slope) parameters were evaluated under the $2 \mathrm{PL}$ model ${ }^{23}$. Test 
information was determined by the summation of all the item information at each value of the underlying construct (i.e., knowledge of whole-genome sequencing). Local dependency analysis was conducted using the Yen's Q3 method of correlated residuals. ${ }^{24}$ The item goodness of fit was assessed using an item fit test statistic. The S- $X^{2}$ likelihood based goodness of fit index was used for detecting item misfits. ${ }^{25}$ In terms of evaluating model fit, the $M_{2}$ statistic ${ }^{26}$ was chosen alongside four additional fit indices: the comparative fit index (CFI), the Tucker-Lewis index (TLI), the root-mean-square error of approximation (RMSEA) and the standardised root mean square residual (SRMR $)^{27}$

\subsection{Statistical analyses}

The proportion of 'correct' responses for each of the 17 items at T1 was described.

Participants were then divided into two groups: (a) students and staff; (b) patients. Chisquare tests were used to examine whether there were differences in responses to each of the 17 items at T1 between the two groups of respondents. The proportion of 'correct' responses was then described for each of the 17 items at T1 and T2 among the subset of respondents who were asked to complete the questionnaire twice, i.e. the students/staff who completed the questionnaire before and after attending a lecture on whole-genome sequencing. Wilcoxon Signed Ranks Test was used to compare responses at T1 vs T2 for each item. Finally, total KOGS scores were calculated for each participant by summing the final set of items produced from the psychometric analyses. Mean KOGS scores were compared at T1 vs T2 among the subset of students/trainees who attended the lectures using non-parametric Wilcoxon Signed Ranks Tests. All statistical analyses were conducted using SPSS v22.

\section{RESULTS}

\section{Sample characteristics}


Of 267 individuals invited to complete the KOGS at T1, a total of 24 participants had incomplete data and were excluded, leaving a final sample size of 243 for the main psychometric and T1 analyses. This comprised 189 students/staff, and 54 patients who completed the survey after they had given informed consent to take part in the 100,000 Genomes Project.

Of the 189 students/trainees, 131 had complete data for the pre- vs post-lecture (T1 vs T2) comparison analyses.

\section{Psychometrics}

Psychometric analyses were conducted on the sample of 243 participants who had complete data at T1. The initial results of the KMO showed that item 5 ("A person shares more of their DNA with their family members than they do with other people") exhibited a KMO index of 0.39; this item was therefore removed. The second iteration of the KMO showed that the overall $\mathrm{KMO}$ index was 0.85 and the lowest $\mathrm{KMO}$ index for the items was 0.64 (item 7, "Whole-genome sequencing can be done on a blood sample"), all of which are above the proposed cut-off KMO value, indicating that the correlation matrix was appropriate for factoring procedures (see Table S1).

In the initial EFA procedures, four items $(1,6,7$ and 10) exhibited factor loadings of less than 0.5 and were removed from further analysis. The result from the final EFA analysis showed that the remaining 12 items achieved factor loadings of greater than 0.5 . These items were considered significant in defining the latent factor (see Table S2).

The averages of the eigenvalues and the eigenvalues of the first five factors for each of the observed, simulated and resampled data indicated that a four factors solution should be retained given that the eigenvalues were greater than the average of the simulated 
eigenvalues (see Table S3). However, the eigenvalue of the first factor being approximately six times more than the second factor indicated a dominance of a single general factor (Figure S1). Moreover, the resulting eigenvalues for the second to fourth factors were not too different from those produced by simulated data (Table S3). A single factor solution accounted for $53 \%$ of the variance while a two-factor solution merely increased the explained variance to $63 \%$. Thus, including a second factor may lead to the development of unnecessary complex theories which have little theoretical value. ${ }^{28}$ Therefore, it was decided that a single factor solution should be included in the model for further analysis.

MSA indicated that the items conformed to a single unidimensional factor and that the item $\mathrm{Hi}$ for all the items were greater than 0.3 (Table S4). The Loevinger's $\mathrm{H}$ coefficient for the scale was at 0.39 , suggesting that acceptable scalability was achieved.

Item fit analysis results indicated that item $15\left(S-X^{2}=19.53(7), p<0.01\right)$ did not fit the model and was removed from further analysis. A second iteration on the item fit analysis did not identify new misfitting items (Table S5).

Local dependency was apparent between item 8 and item $11(r=0.22)$, and item 9 and item $11(r=0.31)$. Both items 8 and 9 had residual correlations of greater than 0.2 with item 11 , indicating that the items were locally related even after the effect of the underlying construct was conditioned out. Item 11 was therefore eliminated to ensure that the assumption of local independence was not violated. Local independence was subsequently achieved with the resulting set of 10 items. However, upon inspection of the item content, item 14 ("Wholegenome sequencing always provides a person with meaningful information about their health") was an inverse duplicate of item 17 ("Whole-genome sequencing may not provide a person with any meaningful information about their health'). Because item $17(b=-0.60)$ was slightly more difficult than item $14(b=-0.61)$, item 14 was considered redundant and removed. 
The item discrimination and the difficulty parameter estimates for the nine items are in Table 1. Regarding model fit, the $M_{2}$ statistics was $46.56(27)$ with a $p$-value $<0.05$. The RMSEA was at $0.05(95 \% \mathrm{Cl} 0.03-0.08)$, SRMR was at 0.06 , and the comparative indices (CFI is $0.97, \mathrm{TLI}$ is 0.97 ) were above the recommended cut-off criteria. The result of the fit indices indicated that an excellent model fit to the data was achieved.

The peak of the test information was 6.23 , which is at the theta level of approximately -.97 (see Figure S2). A downward trend was observed as the theta spread across both ends of the ability (theta) spectrum. The result is unsurprising since there were only nine items and the item discrimination and difficult estimates were relatively close to each other. The internal consistency (Cronbach's alpha) of the scale was 0.79 .

\section{Statistical analyses}

\subsection{T1 analysis results}

As Table 2 shows, the hardest of the 17 items were "A person's genome is the $1 \%$ of their DNA that makes proteins" and "Whole genome sequencing involves looking at most of the DNA in a genome" (both $58.8 \%$ correct overall). The easiest was "A person shares more of their DNA with their family members than they do with other people" ( $90.1 \%$ correct overall). As Table 2 also shows, three items were significantly different $(p<0.001)$ between students/staff and patients: A person's genome is the $1 \%$ of their DNA that makes proteins (students/staff vs patients: $65.6 \%$ vs $35.2 \%$ respectively, $\mathrm{X}^{2}(1)=16.05, \mathrm{p}=0.000062$ ); $A$ person's genome is the complete set of cells in their body (students/staff vs patients: $80.4 \%$ vs $\left.40.7 \%, \mathrm{X}^{2}(1)=32.53, \mathrm{p}=1.17 \times 10^{-8}\right)$; and Scientists know what all parts of the genome do (students/staff vs patients: $90.5 \%$ vs $\left.66.7 \%, X^{2}(1)=18.87, p=0.000014\right)$. 


\subsection{T1 vs T2 (pre-post) comparison results}

When the responses of the students/staff $(n=131)$ were compared between before vs after attending a genomics lecture, the item that had the greatest increase was "Whole-genome sequencing may not provide a person with any meaningful information about their health": correct responses for this item increased from $66.4 \%$ to $87.8 \%$. See Table 3 for this and all comparisons between before-lecture and after-lecture individual item scores.

\subsection{Final 9-item KOGS}

The mean score for the final 9-item version of the KOGS in the sample over all was 6.60 (where $0=$ low knowledge and $10=$ high knowledge). As shown in Table 4, mean scores for patients were significantly lower than those for students/staff (5.39 vs 6.95 respectively, $\mathrm{p}<0.001)$. Among students/staff who completed the questionnaire twice, scores on the final 9-item version of the KOGS significantly increased from before to after attending the genomics lecture (6.92 vs $7.76, p=0.000016)$. The final version of the 9 -item KOGS is shown in Box 1.

\section{DISCUSSION AND CONCLUSION}

\section{1) Discussion}

As part of a larger project evaluating patients' experiences and informed choices about whole-genome sequencing, we have developed a robust 9-item Knowledge of Genome Sequencing (KOGS) questionnaire using a rigorous psychometric measure development approach. The development of the KOGS is very timely as there are several large genomic studies involving patients and the public ongoing in the UK, ${ }^{29,30}$ the $\mathrm{USA}^{31,32}$ and elsewhere ${ }^{33}$ and patient-reported measures regarding whole-genome sequencing are urgently needed. ${ }^{1}$ 
This new whole-genome sequencing knowledge measure is an advance on previous measures because it includes both true and false statements (whereas all the items in Kaphingst et $\mathrm{al}^{3}$ are 'true'), and because the focus is specifically on whole-genome sequencing (whereas the items in Langer et $\mathrm{al}^{6}$ focus on whole-exome sequencing). In addition, no previous measure has, to our knowledge, specifically included measurement of knowledge of what a 'genome' is. A further advantage is that the KOGS is designed to be applicable to a range of stakeholders, including patients, non-genetics healthcare providers and trainees, and the public.

Over the next decade, many millions of individuals will have their genomes sequenced for clinical diagnostic purposes, ${ }^{34}$ with informed consent being required prior to sequencing. For this to be done effectively individuals need to have adequate knowledge. ${ }^{2}$ While defining 'adequate' remains challenging, it is arguably a minimum standard that individuals should have a basic understanding of what the term 'whole-genome sequencing' means, what a 'genome' is, and that there is some degree of uncertainty around the information arising from whole-genome sequencing. While this can be assessed qualitatively, quantitative measures have the advantage that they can be administered on a large-scale to large numbers of individuals, and scored quickly and easily. ${ }^{35}$ The KOGS includes five items on what a genome is and what whole-genome sequencing is, and four on limitations and uncertainty regarding whole-genome sequencing. When administered in the research context the measure was easily administered and acceptable to individuals consenting to having whole-genome sequencing done.

As genomic technologies are embedded in mainstream clinical practice, health care providers who are not expert in genetics will need to discuss whole-genome sequencing with their patients. ${ }^{36}$ The KOGS was found to be effective in demonstrating increases in knowledge after whole-genome sequencing training for non-genetic specialists, and could 
therefore potentially be used as part of a battery of tools to assess clinicians' knowledge in the future.

Although the KOGS was not administered in a survey of the general public in this study, it has been designed as a 'context-neutral' measure (in contrast to, for example, knowledge of having whole-genome sequencing done for the clinical purpose of diagnosis a rare disease), and so would be appropriate to include in a public survey on whole-genome sequencing. Ensuring public trust is critical to large-scale scientific endeavours ${ }^{37}$ and, although the relationship between trust and knowledge is not straightforward, ${ }^{38}$ an easily-administered measure of whole-genome sequencing knowledge may be a valuable resource as part of a larger set of tools for those working to understand public opinion and views of whole-genome sequencing. It also has potential value to assess change in public knowledge and understanding of whole-genome sequencing over time, or to evaluate the effectiveness of public education interventions, such as the online educational animations about wholegenome sequencing developed by Genomics England ${ }^{39}$ and others. ${ }^{40}$

The main limitations of this study are that the 9-item version of the KOGS has only been evaluated in the context of a research study and has yet to be evaluated in a large study assessing content validity. In addition it has not yet been administered to members of the general public. These will be useful directions for future work.

\section{2) Conclusions}

In conclusion, using a rigorous psychometric measure development approach, we have developed a brief, reliable measure of knowledge of whole-genome sequencing, the KOGS.

\section{3) Practice implications}

The KOGS should be useful for researchers and clinicians using whole-genome sequencing with patients and other stakeholders in a variety of contexts. 


\section{Acknowledgements}

This manuscript presents independent research funded by the National Institute for Health Research (NIHR) under the Research for Patient Benefit funding stream (PB-PG-101435016: A study to define patient priorities and preferences when consenting to whole genome sequencing to ensure informed choice). LSC is partially funded by the NIHR Biomedical Research Centre at Great Ormond Street Hospital. The views expressed are those of the authors and not necessarily those of the NHS, the NIHR or the Department of Health. 


\section{REFERENCES}

1. Gray SW, Martins Y, Feuerman LZ, Bernhardt BA, Biesecker BB, Christensen KD, et al. Social and behavioral research in genomic sequencing: approaches from the Clinical Sequencing Exploratory Research Consortium Outcomes and Measures Working Group. Genet Med. 2014;16(10):727-35.

2. Marteau TM, Dormandy E, Michie S. A measure of informed choice. Health Expect. 2001;4(2):99-108.

3. Kaphingst KA, Facio FM, Cheng MR, Brooks S, Eidem H, Linn A, et al. Effects of informed consent for individual genome sequencing on relevant knowledge. Clin Genet. 2012;82(5):408-15.

4. Vassy JL, Lautenbach DM, McLaughlin HM, Kong SW, Christensen KD, Krier J, et al. The MedSeq Project: a randomized trial of integrating whole genome sequencing into clinical medicine. Trials. 2014;15:85.

5. Sanderson SC, Suckiel SA, Zweig M, Bottinger EP. Development and preliminary evaluation of an online educational video about whole-genome sequencing for research participants, patients, and the general public. Genet Med. 2015;18(5):501-12.

6. Langer MM, Roche MI, Brewer NT, Berg JS, Khan CM, Leos C, et al. Development and validation of a genomic knowledge scale to advance informed decision-making research in genomic sequencing. MDM Policy. 2017;2(1):2381468317692582.

7. Blanchette PS, Spreafico A, Miller FA, Chan K, Bytautas J, Kang S, et al. Genomic testing in cancer: patient knowledge, attitudes, and expectations. Cancer. 2014;120(19):3066-73.

8. Furr LA, Kelly SE. The Genetic Knowledge Index: developing a standard measure of genetic knowledge. Genet Test. 1999;3(2):193-9.

9. Henneman L, Timmermans DR, van der Wal G. Public experiences, knowledge and expectations about medical genetics and the use of genetic information. Community Genet. 2004;7(1):33-43.

10. Griffin BH, Chitty LS, Bitner-Glindzicz M. The 100000 Genomes Project: What it means for paediatrics. Arch Dis Child Educ Pract. 2017;102(2):105-7.

11. Genetic Alliance UK Patient Charter 2015. Genome sequencing: what do patients think? Available at: https://www.geneticalliance.org.uk/media/1924/patient-charter-genomesequencing-what-do-patients-think.pdf. Accessed January 24, 2018.

12. British Society for Genetic Medicine 2013. Delivering the 100,000 Genomes Project in the NHS. Available at:

http://www.bsgm.org.uk/media/688955/bsgm_100000_genome_strategy_report.pdf. Accessed January 24, 2018.

13. Ayuso C, Millan JM, Mancheno M, Dal-Re R. Informed consent for whole-genome sequencing studies in the clinical setting. Proposed recommendations on essential content and process. Eur J Hum Genet. 2013;21(10):1054-9. 
14. Genomics England. Participant Information Sheets and Consent Forms. Available at: https://www.genomicsengland.co.uk/taking-part/patient-information-sheets-and-consentforms/. Accessed January 24, 2018.

15. Loe BS, Stillwell D, Gibbons C. Computerized Adaptive Testing provides reliable and efficient depression measurement using the CES-D Scale. J Med Internet Res.

2017;19(9):e302.

16. Holgado-Tello FP, Chacón-Moscoso,S., Barbero-García,I., Vila-Abad,E. Polychoric versus Pearson correlations in exploratory and confirmatory factor analysis of ordinal variables. Quality \& Quantity. 2010;44(1):14.

17. Kirk DB. On the numerical approximation of the bivariate normal (tetrachoric) correlation coefficient. Psychometrika. 1973;38(2):9.

18. Kaiser HF. An index of factorial simplicity. Psychometrika. 1974;39(1):6.

19. Glorfeld LW. An improvement on Horn's parallel analysis methodology for selecting the correct number of factors to retain. Educ Psychol Meas. 1995;55(3):16.

20. Mokken RJ. A theory and procedure of scale analysis: with applications in political research. (Volume 1);1971.

21. Van Schuur WH. Mokken scale analysis: Between the Guttman scale and parametric item response theory. Political Analysis. 2003;11(2):34.

22. Yang FM, Kao ST. Item response theory for measurement validity. Shanghai Arch Psychiatry. 2014;26(3):171-7.

23. Handbook of modern item response theory. Springer Science \& Business Media, 2013.

24. Yen WM. Scaling performance assessments: Strategies for managing local item dependence. J Educ Meas. 1993;30(3):26.

25. Orlando MT, D. Likelihood-based item-fit indices for dichotomous item response theory models. Applied Psychological Measurement. 2000;24(1):15.

26. Maydeu-Olivares AJ, H. Limited information goodness-of-fit testing in multidimensional contingency tables. Psychometrika. 2006;71(4):19.

27. Hu LTB, P.M. Cutoff criteria for fit indexes in covariance structure analysis: Conventional criteria versus new alternatives. Structural Equation Modeling. 1999;6(1):55.

28. Comrey ALL, H.B. A first course in factor analysis. Psychology Press, 2013.

29. UK Biobank. Available at: http://www.ukbiobank.ac.uk. Accessed January 24, 2018.

30. East London Genes and Health. Health ELG. East London Genes \& Health website homepage. Available at: http://www.genesandhealth.org/. Accessed January 24, 2018.

31. National Institutes of Health (NIH) All of Us Research Program. Available at: https://allofus.nih.gov/. Accessed January 24, 2018.

32. The eMERGE (Electronic Medical Records and Genomics) Network. Available at: https://emerge.mc.vanderbilt.edu/. Accessed 24 January, 2018. 
33. Global Alliance for Genomics \& Health (GA4GH). Available at: https://www.ga4gh.org/. Accessed 24 January, 2018.

34. Birney E, Vamathevan J, Goodhand P. Genomics in healthcare: GA4GH looks to 2022. bioRxiv. 2017.

35. Marks DF. Questionnaires and Surveys. In: Marks DFY, L., editor. Research Methods for Clinical and Health Psychology. London: Sage, 2004.

36. Feero WG, Manolio TA, Khoury MJ. Translational research is a key to nongeneticist physicians' genomics education. Genet Med. 2014;16(12):871-3.

37. Tutton R, Kaye J, Hoeyer K. Governing UK Biobank: the importance of ensuring public trust. Trends Biotechnol. 2004;22(6):284-5.

38. Sturgis $P$, Allum N. Science in Society: Re-evaluating the deficit model of public attitudes. Public Underst Sci. 2004;13(1):55-74.

39. Genomics England infographics and videos. Available at:

https://www.genomicsengland.co.uk/the-100000-genomes-project/understanding-genomics/. Accessed 24 January, 2018.

40. Sanderson SC, Suckiel SA, Zweig M, Bottinger EP. Development and preliminary evaluation of an online educational video about whole-genome sequencing for research participants, patients, and the general public. Genet Med. 2016;18(5):501-12. 